\section{Características da assistência ao trabalho de parto e parto em três modelos de atenção no SUS, no Município de Belo Horizonte, Minas Gerais, Brasil}

\author{
Characteristics of labor and delivery care in three \\ healthcare models within the Unified National \\ Health System in Belo Horizonte, \\ Minas Gerais State, Brazil
}

.

\section{Abstract}

This cross-sectional study of 831 low-risk pregnancies compared the management of labor and delivery in a birthing center, a hospital that had previously won the "Galba de Araújo" Award (for excellence in obstetric and neonatal care), and a standard-protocol maternity facility. The rates for use of ocytocin during labor were $27.9 \%, 59.5 \%$, and $40.1 \%$, while amniotomy was performed in $67.6 \%, 73.6 \%$, and $82.2 \%$ of the women, respectively. Episiotomy rates were lower in the first two facilities, which have adopted patient-centered obstetric practices $(7.2 \%$ at the birthing center and $14.8 \%$ at the award-winning hospital) as compared to $54.9 \%$ at the standard maternity facility. The liberal offer of epidural anesthesia at the awarding-winning hospital resulted in a higher anesthesia rate (54.4\%) as compared to the standard facility (7.7\%). Forceps delivery and neonatal admission rates were higher in the standard hospital, but there were no differences in mean Apgar or cesarean rates. The findings suggest resistance to selective use of interventions in all three models of obstetric care, although favoring the birthing center as a strategy for controlling interventions during labor and childbirth in low-risk pregnancies, with no resulting harm to the mothers or newborns.

Humanizing Delivery; Obstetric Labor; Maternal and Child Health; Health Services Research
Sibylle Emilie Vogt 1,2

Simone Grilo Diniz 3

Carlos Mendes Tavares 3

Nagela Cristine Pinheiros Santos 2

Camilla Alexsandra Schneck 4

Bianca Zorzam 3

Débora de Andrade Vieira 4

Kátia Silveira da Silva 5

Marcos Augusto Bastos Dias 5

\section{Introdução}

A assistência obstétrica no Brasil é caracterizada pelo emprego inapropriado de intervenções no processo fisiológico do trabalho de parto, cujo uso abusivo pode levar a efeitos danosos para mãe e filho $1,2,3$. Além de desconfortos físicos para a mulher e dos riscos associados às altas taxas de partos cirúrgicos, de episiotomias e do uso rotineiro da ocitocina e da amniotomia, acumulamse evidências sobre uma epidemia de prematuridade tardia causada pela interrupção eletiva e sem indicação clínica precisa da gravidez 4,5 .

A taxa de cesárea, indicador importante do modelo de atenção chamado de "tecnicista" que está em vigor no Brasil 1,2,6, alcançou em 2006 uma média nacional de 43,8\% 7. Essa tendência foi concomitante ao aumento da prematuridade que variou entre $3,4 \%$ e $15 \%$ em diferentes regiões do país ${ }^{4}$. A episiotomia, outro indicador importante, apresenta média nacional de $71,6 \%$, e estudos no Rio de Janeiro mostraram uso de ocitocina entre $39,3 \%$ e $64,4 \%$ 8,9.

Apesar do acesso praticamente universal ao pré-natal e ao parto hospitalar em todo país e dos avanços importantes no conhecimento científico, observa-se um paradoxo na assistência obstétrica brasileira: as mulheres e seus recém-nascidos adoecem e morrem tanto pela falta quanto pelo uso inapropriado e inseguro da tecnologia 2.

Entendendo-se como modelo de atenção a forma de organização das práticas assistenciais, 
o Ministério da Saúde tem desenvolvido, ao longo das últimas décadas, estratégias para promover um novo modelo de assistência ao parto, que visam à humanização e à redução das intervenções desnecessárias 10. Entre outras estratégias, foram criados os Centros de Parto Normal (CPN) e instituído o Prêmio Galba de Araújo, que valoriza as maternidades que implementam práticas humanizadas. Na última década, houve reestruturação e adaptação à política de humanização em vários serviços no país, mas o modelo tradicional da assistência obstétrica com altas taxas de intervenção ainda é predominante 1,11 .

Para fins desta pesquisa, esses dois modelos assistenciais foram definidos como representantes do modelo humanizado por terem incorporadas práticas baseadas em evidências científicas que favorecem o parto fisiológico. Compreendendo o processo de implementação de práticas humanizadas e baseadas em evidências científicas como um continuum dinâmico e ainda em construção em nosso país, podemos afirmar que se situam, em um dos extremos, os CPN como modelo menos intervencionista e mais humanizado e, no extremo oposto, os hospitais sem incorporação dessas práticas no cotidiano assistencial. Os hospitais premiados ocupariam um lugar intermediário nesse continuum.

O presente estudo faz parte de uma pesquisa denominada Resultados da Assistência ao Parto de Gestantes de Baixo Risco em Hospitais "Galba" e Hospitais "Prevalentes": um Estudo Comparati$v o$, realizada em São Paulo e Belo Horizonte (Minas Gerais), por pesquisadores da Faculdade de Saúde Pública, Universidade de São Paulo e colaboradores e financiada pelo Ministério da Saúde. Ele tem como objetivo avaliar a frequência das intervenções sobre o trabalho de parto de mulheres de baixo risco nos três modelos assistenciais definidos. Nossa hipótese é que, nos serviços com incorporação de práticas humanizadas, a frequência de intervenções para a condução do trabalho de parto seja menor.

\section{Métodos}

Trata-se de um estudo transversal, realizado com dados de prontuários referentes à assistência ao trabalho de parto de mulheres de baixo risco atendidas em 2006 em três serviços: um hospital representativo do modelo assistencial vigente (HP - Hospital Prevalente), uma maternidade vencedora do Prêmio Galba de Araújo (HG - Hospital Galba) e um CPN peri-hospitalar, todos situados em Belo Horizonte. Os critérios de inclusão foram: idade gestacional entre 37 e 42 semanas, gestação única, apresentação cefálica, ausência de intercorrências na gravidez e comorbidades e início de trabalho de parto espontâneo. Esses critérios também definem as condições exigidas para admissão no CPN.

Após estudo piloto, o cálculo da amostra se baseou na prevalência da episiotomia de 50\% no grupo atendido no HG e uma diferença de 15 pontos percentuais para menos no grupo atendido no CPN (35\%). Considerando um alfa de 0,05 (monocaudal), poder de teste de 0,95 e razão de 1:1 entre o grupo de estudo e o grupo controle, obtém-se um tamanho da amostra de 232 prontuários. Acrescido de $20 \%$ para reposição antecipada de eventuais perdas, a amostra totalizou aproximadamente 277 indivíduos para cada grupo. Para o cálculo, foi utilizado o programa Power and Sample Size Calculation version 3.0 (Dupont WD, Plummer WD. Department of Biostatistics, Vanderbilt University School of Medicine, Nashville, Estados Unidos. http://biostat. mc.vanderbilt.edu/wiki/Main/PowerSample Size). A episiotomia foi escolhida como variável para cálculo da amostra, por ser um importante indicador das práticas obstétricas dos serviços e ter registro preciso no prontuário 2,3,12.

Foram sorteados 277 prontuários por amostragem aleatória simples e sistemática entre o total das mulheres atendidas para a assistência ao parto em cada serviço, correspondendo a 8.611, 933 e 4.809 prontuários do HG, do CPN e do HP, respectivamente. Como variáveis de desfecho foram selecionados os usos da ocitocina, amniotomia, analgesia e da episiotomia. Tais variáveis desempenham importante papel na "cascata de intervenções" e nos riscos associados e são utilizadas na literatura para a discussão dessa temática 2,3,13,14,15. Ainda foram coletados dados sobre características sociodemográficas e obstétricas das mulheres, o tipo de parto e sobre resultados neonatais.

Os dados foram extraídos dos prontuários mediante consulta à folha da evolução clínica e do partograma, se existente, para obter informações sobre a dilatação na admissão, o tipo de parto, a realização da episiotomia e da amniotomia. $\mathrm{O}$ uso da ocitocina foi verificado pela checagem da droga pela enfermagem na folha da prescrição e a analgesia pela presença da folha do anestesista, obrigatória nas duas maternidades. Dados sociodemográficos, assim como o número de consultas, foram extraídos da Declaração de Nascidos Vivos, e os dados sobre o recém-nascido da folha do pediatra.

O HG, único hospital premiado existente em Belo Horizonte, é uma instituição filantrópica. A maternidade do HG atende tanto mulheres de risco habitual quanto as de alto risco, oferecendo amplamente métodos farmacológicos e não 
farmacológicos para o manejo da dor do parto. A assistência ao parto é realizada por uma equipe composta por médicos e enfermeiras obstetras.

O CPN opera como um centro de parto peri-hospitalar do HG. No pronto atendimento do HG, que é o setor de entrada comum tanto para as mulheres que serão atendidas no HG quanto para aquelas que optam para o CPN, a sua assistência é oferecida a todas as mulheres de risco habitual, que cumprem o protocolo da instituição e não pretendem usar analgesia medicamentosa. Em caso de evolução de distocia do trabalho de parto ou para atender ao pedido por analgesia, que surgiu posteriormente à admissão durante a progressão do trabalho de parto, a mulher é transferida para a maternidade. O CPN dispõe da possibilidade do parto na água e há amplo uso de métodos não farmacológicos para o alívio da dor.

O hospital representativo do modelo aqui chamado de HP foi selecionado por indicação de um membro da Secretaria Municipal de Saúde familiarizado com o contexto local. É um hospital público com título de Maternidade Segura e oferece residência médica em obstetrícia e pediatria. Em 2006, o serviço dispunha de chuveiro e área para deambular como métodos não farmacológicos de alivio da dor, além da analgesia peridural. A assistência ao trabalho de parto e parto é prestada por residentes em obstetrícia supervisionados pelo preceptor.

A coleta dos dados foi realizada por membros da pesquisa, por meio de um instrumento pré-definido, entre junho e dezembro de 2008, após aprovação do projeto nos Comitês de Ética em Pesquisa das três instituições envolvidas. A entrada dos dados no banco foi feita com dupla digitação. Na análise, após a validação, os dados ignorados remanescentes de cada variável não foram considerados. As diferenças entre as frequências foram analisadas por meio do teste quiquadrado de Pearson e Fisher, quando indicado.

\section{Resultados}

Foram analisados 277 prontuários em cada serviço com perda de informação diferenciada para cada variável e cada modelo entre $0,5 \%$ (valor de Apgar no 5o minuto) e 4,3\% para o uso de ocitocina e $4,2 \%$ para a variável dilatação na admissão, com exceção do registro da rotura artificial de membranas, que apresentou um percentual de $16,3 \%$ de reposta ignorada.

A Tabela 1 mostra a frequência das características sociodemográficas e as condições clínicas das parturientes. Tendo em conta o total de prontuários analisados nos três serviços, o percentual da gravidez na adolescência foi de 24,7\%. O nível de escolaridade da clientela também se mostra semelhante: a maioria das mulheres frequentou a escola de oito a 11 anos nos três serviços. No CPN e no HG, foram atendidas mais mulheres com situação conjugal estável do que no HP.

Considerando as variáveis obstétricas, são encontradas diferenças importantes entre os três modelos assistenciais referentes à parição, número de consultas de pré-natal, estado de membranas amnióticas e dilatação cervical à admissão. A cobertura do pré-natal foi $100 \%$ em todos os três serviços, mas quase $7 \%$ das mulheres do total da população estudada tiveram no máximo três consultas.

O HG atendeu a um maior percentual de primíparas e de mulheres com menor número de consultas pré-natais, enquanto o CPN assistiu menos primíparas e mulheres com sete ou mais consultas. Mais da metade das mulheres admitidas no HG estava com menos de $5 \mathrm{~cm}$ de dilatação cervical e quase um terço já internou com as membranas amnióticas rotas.

A Tabela 2 lista a frequência das características da assistência segundo os três modelos. O HG apresentou o maior percentual de uso de ocitocina $(59,5 \%)$ e da analgesia peridural $(54,4 \%)$. Todos os serviços utilizaram a amniotomia, como meio de conduzir o trabalho de parto num grande contingente de mulheres com membranas íntegras na admissão, resultando num percentual médio, entre essas mulheres, de $74,1 \%$. No CPN foram encontradas menores frequências no uso de ocitocina, de amniotomia e de episiotomia, com percentuais iguais a $27,9 \%, 67,6 \%$ e $7,2 \%$, respectivamente. Foi construído um indicador composto para resumir as intervenções utilizadas constituído pelas variáveis: parto normal, uso de analgesia, episiotomia, ocitocina e amniotomia. Cerca de um quarto das mulheres do CPN não foi submetido a nenhuma intervenção $(25,6 \%)$, enquanto no HG o percentual foi de $14,3 \%$ e no HP 5,3\%.

A taxa de cesárea foi praticamente igual para o HG e o HP, enquanto o parto a fórceps ocorreu com maior frequência no HP $(9,4 \%)$ (Tabela 2$)$. Cabe ressaltar que não fizeram parte da amostra as mulheres transferidas por terem optado pela analgesia do parto após sua admissão no CPN e as que foram transferidas por outros motivos e submetidas à cesárea ou ao parto fórceps no HG. Somente dois recém-nascidos do total da amostra receberam um escore de Apgar menor do que 7 no 5 o minuto de vida, e o percentual de internação em unidade neonatal foi menor no CPN e no HG do que no HP.

A Tabela 3 mostra a comparação das intervenções utilizadas no trabalho de parto entre o 
Aspectos sociodemográficos e obstétricos das mulheres atendidas segundo o modelo assistencial: Centro de Parto Normal (CPN), Hospital Galba (HG) e Hospital Prevalente (HP). Belo Horizonte, Minas Gerais, Brasil, 2006.

\begin{tabular}{|c|c|c|c|c|c|c|c|c|}
\hline \multirow[t]{3}{*}{ Características } & \multicolumn{6}{|c|}{ Modelos assistenciais } & \multicolumn{2}{|c|}{ Total } \\
\hline & \multicolumn{2}{|c|}{ CPN } & \multicolumn{2}{|c|}{ HG } & \multicolumn{2}{|c|}{ HP } & \multirow[b]{2}{*}{$n$ * } & \multirow[b]{2}{*}{$\%$} \\
\hline & $n$ * & $\%$ & $n *$ & $\%$ & $n$ * & $\%$ & & \\
\hline \multicolumn{9}{|l|}{ Sociodemográficas } \\
\hline \multicolumn{9}{|l|}{ Idade (anos) } \\
\hline$\leq 19$ & 59 & 21,8 & 74 & 27,3 & 65 & 25,1 & 198 & 24,7 \\
\hline $20-34$ & 198 & 73,1 & 184 & 67,9 & 189 & 73,0 & 571 & 71,3 \\
\hline$\geq 35$ & 14 & 5,2 & 13 & 4,8 & 5 & 1,9 & 32 & 4,0 \\
\hline \multicolumn{9}{|l|}{ Escolaridade (anos) } \\
\hline $0-7$ & 89 & 32,7 & 84 & 30,7 & 89 & 33,3 & 262 & 32,2 \\
\hline $8-11$ & 169 & 62,1 & 172 & 62,8 & 155 & 58,1 & 496 & 61,0 \\
\hline$>11$ & 14 & 5,1 & 18 & 6,6 & 23 & 8,6 & 55 & 6,8 \\
\hline \multicolumn{9}{|l|}{ Situação conjugal } \\
\hline Com relação estável & 117 & 42,2 & 85 & 30,8 & 63 & 23,5 & 265 & 32,3 \\
\hline Sem relação estável & 160 & 57,8 & 191 & 69,2 & 205 & 76,5 & 556 & 67,7 \\
\hline \multicolumn{9}{|l|}{ Obstétricas } \\
\hline \multicolumn{9}{|l|}{ Parição } \\
\hline Primípara & 101 & 36,7 & 167 & 60,3 & 134 & 49,3 & 402 & 48,8 \\
\hline Multípara & 174 & 63,3 & 110 & 39,7 & 138 & 50,7 & 422 & 51,2 \\
\hline \multicolumn{9}{|l|}{ Consulta pré-natal } \\
\hline $1-3$ & 14 & 5,1 & 26 & 9,7 & 16 & 6,0 & 56 & 6,9 \\
\hline $4-6$ & 146 & 53,7 & 114 & 42,5 & 111 & 41,4 & 371 & 45,9 \\
\hline$\geq 7$ & 112 & 41,2 & 128 & 47,8 & 141 & 52,6 & 381 & 47,2 \\
\hline \multicolumn{9}{|l|}{ Dilatação cervical $(\mathrm{cm})$ ** } \\
\hline$<5$ & 101 & 38,8 & 135 & 51,1 & 92 & 33,8 & 328 & 41,2 \\
\hline$\geq 5$ & 159 & 61,2 & 129 & 48,9 & 180 & 66,2 & 468 & 58,8 \\
\hline \multicolumn{9}{|l|}{ Membranas amnióticas ** } \\
\hline Rotas & 42 & 15,2 & 60 & 22,9 & 51 & 18,8 & 153 & 18,9 \\
\hline Íntegras & 234 & 84,8 & 202 & 77,1 & 220 & 81,2 & 656 & 81,1 \\
\hline
\end{tabular}

* O n amostral de cada variável não inclui seus valores ignorados;

** No momento da admissão hospitalar.

CPN e o HP. Foi constatado que no CPN houve frequência menor de uso da ocitocina em primíparas e para aquelas cuja dilatação foi maior que $5 \mathrm{~cm}$. Também foi menor o percentual de uso da amniotomia e da episiotomia tanto para primíparas quanto para multíparas, contudo não houve diferenças significativas entre as duas unidades na realização da amniotomia para as mulheres admitidas com dilatação menor que $5 \mathrm{~cm}$. Chama atenção que nas duas unidades pelo menos dois terços das mulheres foram submetidos à amniotomia. Para a episiotomia foi observado uso no HP 7,6 vezes $(7,2 \%$ versus $54,9 \%)$ maior do que no $\mathrm{CPN}$, sendo a diferença mais acentuada para as multíparas. A internação em unidade neonatal foi muito mais frequente para o HP $(5,4 \%$ versus $1,8 \%)$, ainda que não tenha havido diferença na vitalidade dos recém-nascidos ao nascer, expressa pelo índice de Apgar menor que 7 no 5 o minuto de vida.

A Tabela 4 se refere à comparação entre o CPN e o HG, considerado um modelo no meio do continuum da humanização da assistência com incorporação de métodos não farmacológicos para o alívio da dor na assistência, fusão entre o ambiente do pré-parto e parto e uso do partograma para intervenção adequada, entre outras. O uso da ocitocina foi significativamente menor no CPN, mesmo considerando a parição e a dilatação cervical na admissão. Porém não foram verificadas diferenças significativas quando se analisou o uso da amniotomia. Em relação à 
Intervenções realizadas na assistência ao trabalho de parto e parto segundo o modelo assistencial: Centro de Parto Normal (CPN), Hospital Galba (HG) e Hospital Prevalente (HP). Belo Horizonte, Minas Gerais, Brasil, 2006.

\begin{tabular}{|c|c|c|c|c|c|c|c|c|}
\hline & \multicolumn{6}{|c|}{ Modelos assistenciais } & \multicolumn{2}{|c|}{ Total } \\
\hline & \multicolumn{2}{|c|}{ CPN } & \multicolumn{2}{|c|}{ HG } & \multicolumn{2}{|c|}{ HP } & \multirow[b]{2}{*}{$n$ * } & \multirow[b]{2}{*}{$\%$} \\
\hline & $n$ * & $\%$ & $n$ * & $\%$ & $n$ * & $\%$ & & \\
\hline Ocitocina & 69 & 27,9 & 163 & 59,5 & 110 & 40,1 & 342 & 43,0 \\
\hline Amniotomia & 140 & 67,6 & 128 & 73,6 & 143 & 82,2 & 411 & 74,1 \\
\hline Analgesia ** & - & - & 149 & 54,4 & 21 & 7,7 & 170 & 31,1 \\
\hline Episiotomia & 20 & 7,2 & 41 & 14,8 & 152 & 54,9 & 213 & 25,6 \\
\hline \multicolumn{9}{|l|}{ Tipo de parto } \\
\hline Cesárea & - & - & 13 & 4,7 & 12 & 4,3 & 25 & 3,0 \\
\hline Fórceps & - & - & 3 & 1,1 & 26 & 9,4 & 29 & 3,4 \\
\hline Espontâneo & 277 & 100,0 & 261 & 94,2 & 239 & 86,3 & 777 & 93,6 \\
\hline Assistência sem intervenção *** & 61 & 25,6 & 37 & 14,3 & 13 & 5,3 & 111 & 15,0 \\
\hline Apgar $<7 \#$ & 0 & 0,0 & 1 & 0,4 & 1 & 0,4 & 2 & 0,2 \\
\hline Internação em unidade neonatal \#\# & 5 & 1,8 & 6 & 2,2 & 14 & 5,1 & 25 & 3,0 \\
\hline
\end{tabular}

* O n amostral de cada variável não inclui seus valores ignorados;

** Analgesia peridural com dose única;

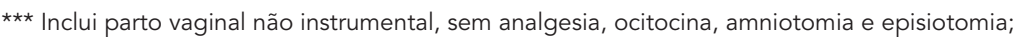

\# No 5o minuto de vida;

\#\# Unidades de terapia intensiva e intermediária.

episiotomia, foi identificado um percentual menor do procedimento no CPN, mas a diferença não se manteve significativa para os subgrupos de parição. Os resultados neonatais foram iguais para ambos os modelos.

A Tabela 5 mostra as diferenças da assistência prestada no HG e no HP. No HP houve uma frequência significativamente menor $(40,2 \%)$ da ocitocina, tanto em primíparas quanto em multíparas, e entre as mulheres com dilatação igual ou maior que $5 \mathrm{~cm}$. Somente quando se compara o uso da ocitocina na ausência de analgesia, a tendência se inverte $(27,4 \%$ no HG versus $37,5 \%$ no HP), no entanto a diferença não é significativa. A comparação do uso da analgesia revela diferenças significativas entre os dois serviços, independentemente da dilatação na admissão. Nas primíparas, o percentual de analgesia chega a $64,8 \%$ no HG versus $12,9 \%$ no HP. A diferença é mais acentuada nas multíparas, dentre as quais o uso de analgesia foi 13 vezes maior no HG. A analgesia foi indicada com maior frequência para as mulheres com menos de $5 \mathrm{~cm}$ de dilatação cervical à admissão, tanto no HG quanto no HP $(63,2 \%$ versus $12,4 \%)$, do que para aquelas com dilatação maior do que $5 \mathrm{~cm}$ ( $46,1 \%$ versus $5,6 \%)$. No HG, a realização da analgesia na presença da ocitocina foi mais do que o dobro do que na sua ausência, enquanto no HP o percentual tripli- ca nesses casos. A episiotomia foi utilizada com maior frequência no HP (54,9\% versus $14,8 \%)$ do que no HG com diferença significativa nas primíparas e multíparas. O mesmo se encontra em relação ao parto a fórceps e à internação em unidade neonatal.

\section{Discussão}

O delineamento do estudo e a heterogeneidade das mulheres nos três modelos em relação à parição e à dilatação na admissão são limitações do estudo, assim como as diferenças na oferta e na disponibilidade da analgesia peridural e da estrutura física do pré-parto/bloco obstétrico entre os serviços. As diferenças na oferta da analgesia devem ter afetado o uso da ocitocina, assim como a estrutura da área física dificultou ou facilitou posições supinas no trabalho de parto, que influenciam a progressão do trabalho de parto e, posteriormente, o emprego de intervenções 3 . O viés da parição pôde ser analisado pela comparação dos desfechos de acordo com sua proporção em cada serviço. A exclusão das transferências na amostra do CPN constituiu um viés de seleção, que pode ter subestimado os resultados no CPN relativo à taxa de intervenções e dos resultados neonatais. 
Comparação das intervenções realizadas na assistência ao parto e trabalho de parto no Centro de Parto Normal (CPN) e no Hospital Prevalente (HP). Belo Horizonte, Minas Gerais, Brasil, 2006.

\begin{tabular}{|c|c|c|c|c|c|}
\hline & \multicolumn{4}{|c|}{ Modelos assistenciais } & \multirow{3}{*}{$\begin{array}{c}\text { Valor de } \\
\qquad p^{\star \star}\end{array}$} \\
\hline & \multicolumn{2}{|c|}{ CPN } & \multicolumn{2}{|c|}{$\mathrm{HP}$} & \\
\hline & $n$ * & $\%$ & $n$ * & $\%$ & \\
\hline Uso de ocitocina & 69 & 27,9 & 110 & 40,1 & 0,003 \\
\hline Primíparas & 30 & 32,3 & 62 & 46,9 & 0,027 \\
\hline Multíparas & 39 & 25,3 & 45 & 32,8 & 0,158 \\
\hline 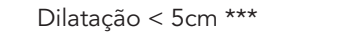 & 38 & 40,4 & 46 & 50,5 & 0,167 \\
\hline Dilatação $\geq 5 \mathrm{~cm}$ *** & 26 & 18,6 & 62 & 34,8 & 0,001 \\
\hline Uso da amniotomia & 140 & 67,6 & 143 & 82,2 & 0,001 \\
\hline Primíparas & 47 & 65,3 & 71 & 82,6 & 0,013 \\
\hline Multíparas & 91 & 68,4 & 68 & 81,9 & 0,028 \\
\hline Dilatação $<5 \mathrm{~cm} * \star \star$ & 56 & 74,7 & 55 & 79,7 & 0,472 \\
\hline 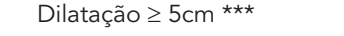 & 78 & 65,5 & 84 & 83,2 & 0,003 \\
\hline Uso da episiotomia & 20 & 7,2 & 152 & 54,9 & $<0,001$ \\
\hline Primípara & 13 & 12,9 & 106 & 79,1 & $<0,001$ \\
\hline Multípara & 7 & 4,0 & 44 & 31,9 & $<0,001$ \\
\hline Assistência sem intervenção \# & 61 & 25,6 & 13 & 5,3 & $<0,001$ \\
\hline Apgar $<7$ \#\# & 0 & 0,0 & 1 & 0,4 & 0,498 \\
\hline Internação neonatal \#\#\# & 5 & 1,8 & 15 & 5,4 & 0,038 \\
\hline
\end{tabular}

* O n amostral de cada variável não inclui seus valores ignorados;

** Método estatístico empregado: qui-quadrado de Pearson ou Fisher Exact;

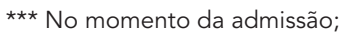

\# Inclui parto vaginal não instrumental, sem analgesia, ocitocina, amniotomia e episiotomia;

\#\# No 5o minuto de vida;

\#\#\# Unidades de terapia intensiva e intermediária.

Como uma provável hipótese para explicar o percentual menor de primíparas na amostra do CPN, pode ser pensada a indisponibilidade de analgesia nessa instituição e a necessidade de transferência para o HG, quando a parturiente a deseja após ter dado entrada no CPN para a assistência ao parto. Essa informação é dada às mulheres na hora da admissão e pode ser decisiva para sua opção entre o CPN e o HG como local do parto. Talvez um maior contingente de primíparas prefira o atendimento no $\mathrm{HG}$, por ter medo de não suportar a dor do parto. Em consequência, o percentual de primíparas no HG é maior. Também foi encontrado, no HG, maior percentual de mulheres admitidas com dilatação menor ou com bolsa rota. Tais condições podem ter levado ao uso de intervenções na condução do trabalho de parto.

A admissão precoce (dilatação menor do que $5 \mathrm{~cm}$ ) deve ser evitada por constituir um fator predisponente para a realização de intervenções desnecessárias e a do parto cirúrgico 3,8,16. A admissão precoce parece prática rotineira nas materni- dades brasileiras, pois no Rio de Janeiro o percentual encontrado foi de $64,6 \% 8$ e em São Paulo de $35,5 \%$ e $43,4 \%$ numa maternidade pública e num CPN peri-hospitalar, respectivamente 12 .

A frequência maior de uso da ocitocina nas mulheres com admissão precoce, observada neste estudo em todos os modelos, indica a importância do momento correto da admissão para evitar intervenções desnecessárias na assistência ao trabalho de parto. O papel da aceleração do trabalho de parto para aumentar o giro dos leitos do pré-parto e a capacidade de atendimento, em caso de superlotação ou de admissão precoce, não pode ser negado, levando a intervenções desnecessárias frequentes 8 . Informação mais adequada no pré-natal sobre os sinais de trabalho de parto e a estruturação da rede com atendimento perto do domicílio seria boa estratégia para evitar a procura precoce das gestantes por vaga e as intervenções para acelerar o trabalho de parto a que serão submetidas.

Embora as taxas encontradas da ocitocina e da amniotomia possam ser consideradas altas 
Comparação das intervenções realizadas na assistência ao parto e trabalho de parto no Centro de Parto Normal (CPN) e Hospital Galba (HG). Belo Horizonte, Minas Gerais, Brasil, 2006.

\begin{tabular}{|c|c|c|c|c|c|}
\hline & \multicolumn{4}{|c|}{ Modelos assistenciais } & \multirow[t]{3}{*}{ Valor de $p$ ** } \\
\hline & \multicolumn{2}{|c|}{ CPN } & \multicolumn{2}{|c|}{ HG } & \\
\hline & $n$ * & $\%$ & $n$ * & $\%$ & \\
\hline Uso de ocitocina & 69 & 27,9 & 163 & 59,5 & $<0,001$ \\
\hline Primíparas & 30 & 32,3 & 109 & 66,1 & $<0,001$ \\
\hline Multíparas & 39 & 25,5 & 54 & 49,5 & $<0,001$ \\
\hline Dilatação < 5cm *** & 38 & 40,4 & 100 & 74,6 & $<0,001$ \\
\hline 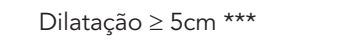 & 26 & 18,6 & 55 & 43,0 & $<0,001$ \\
\hline Uso da amniotomia & 140 & 67,6 & 128 & 73,6 & 0,207 \\
\hline Primíparas & 47 & 65,3 & 86 & 78,2 & 0,055 \\
\hline Multíparas & 91 & 68,4 & 42 & 65,6 & 0,695 \\
\hline Dilatação $<5 \mathrm{~cm}$ *** & 56 & 74,7 & 64 & 71,1 & 0,610 \\
\hline 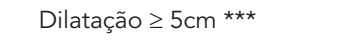 & 78 & 65,5 & 56 & 74,7 & 0,181 \\
\hline Uso da episiotomia & 20 & 7,2 & 41 & 14,8 & 0,004 \\
\hline Primípara & 13 & 12,9 & 37 & 22,2 & 0,059 \\
\hline Multípara & 7 & 4,1 & 4 & 3,6 & 0,869 \\
\hline Assistência sem intervenção \# & 61 & 25,6 & 37 & 14,3 & $<0,001$ \\
\hline Apgar $<7$ \#\# & 0 & 0,0 & 1 & 0,4 & 0,501 \\
\hline Internação neonatal \#\#\# & 5 & 1,8 & 6 & 2,2 & 0,761 \\
\hline
\end{tabular}

* O n amostral de cada variável não inclui seus valores ignorados;

** Método estatístico empregado: qui-quadrado de Pearson ou Fisher Exact;

*** No momento da admissão;

\# Inclui parto vaginal não instrumental, sem analgesia, ocitocina, amniotomia e episiotomia;

\#\# No 5o minuto de vida;

\#\#\# Unidades de terapia intensiva e intermediária.

para todas as modalidades, seu uso foi menos frequente no CPN. Schneck 12 também encontrou frequência menor de uso de ocitocina (23,6\%) no $\mathrm{CPN}$, quando comparou a assistência prestada a 1.315 mulheres de risco habitual num CPN perihospitalar em São Paulo com um hospital vencedor do Prêmio Galba de Araújo (47,2\%).

A utilização da ocitocina durante o trabalho de parto sem o monitoramento adequado da gestante é uma prática perigosa, uma vez que essa droga pode causar riscos importantes para a saúde de mãe e feto. A taquisistolia uterina é frequentemente causada pelo seu uso sem controle adequado e pela falta do acompanhamento rigoroso do bem-estar materno e fetal durante o trabalho de parto. Diante desses fatos, a ocitocina deve ser utilizada com a menor frequência possível 3,13,17. A internação das mulheres na fase ativa do trabalho de parto, a presença de um acompanhante, a possibilidade de deambular e de assumir posições não supinas e o uso da ocitocina restrito ao trabalho de parto diagnosticado como prolongado por meio do partograma são medidas comprovadamente benéficas para o controle de intervenções desnecessárias 3 . Tanto a tendência do seu uso no presente estudo quanto os resultados encontrados por Schneck 12 sugerem o CPN, onde essas condutas são institucionalizadas, como estratégia factível para reduzir a utilização inapropriada dessa droga e seus efeitos adversos.

O uso ainda mais frequente da amniotomia em todas as modalidades de assistência, embora com menores percentuais para o CPN, sugere sua prática rotineira e contrária às evidências científicas 3,18 . Schneck 12 observou prevalências similares entre as mulheres admitidas com bolsa íntegra de $71,3 \%$ no CPN e $84,5 \%$ na maternidade. No Rio de Janeiro, foram encontrados percentuais bem menores, calculados sobre o total das amostras, entre $24,3 \%$ e $42,4 \% 8$ e de $36,2 \%$ e $41,2 \% 9$.

A aceleração do trabalho de parto, seja com ocitocina e/ou com a amniotomia, tem um papel fundamental na "cascata de intervenção" e sobre a redução da taxa de parto espontâneo 14,15,16. 
Comparação das intervenções realizadas na assistência ao parto e trabalho de parto no Hospital Galba (HG) e Hospital Prevalente (HP). Belo Horizonte, Minas Gerais, Brasil, 2006.

\begin{tabular}{|c|c|c|c|c|c|}
\hline & \multicolumn{4}{|c|}{ Modelos assistencias } & \multirow{3}{*}{$\begin{array}{l}\text { Valor de } \\
\qquad p^{* *}\end{array}$} \\
\hline & \multicolumn{2}{|c|}{ HG } & \multicolumn{2}{|c|}{ HP } & \\
\hline & $n$ * & $\%$ & $n$ * & $\%$ & \\
\hline Uso de ocitocina & 163 & 59,5 & 110 & 40,1 & $<0,001$ \\
\hline Primíparas & 109 & 66,1 & 62 & 46,9 & 0,001 \\
\hline Multíparas & 54 & 49,5 & 45 & 32,8 & 0,008 \\
\hline 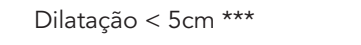 & 100 & 74,6 & 46 & 50,5 & $<0,001$ \\
\hline Dilatação $\geq 5 \mathrm{~cm}$ *** & 55 & 43,0 & 62 & 34,8 & 0,148 \\
\hline Sem presença de analgesia \# & 34 & 27,4 & 93 & 37,5 & 0,053 \\
\hline Na presença de analgesia \# & 127 & 86,4 & 15 & 71,4 & 0,076 \\
\hline Uso da amniotomia & 128 & 73,6 & 143 & 82,2 & 0,053 \\
\hline Primíparas & 86 & 78,2 & 71 & 82,6 & 0,446 \\
\hline Multíparas & 42 & 65,6 & 68 & 81,9 & 0,024 \\
\hline Dilatação $<5 \mathrm{~cm} * \star \star$ & 64 & 71,1 & 55 & 79,7 & 0,216 \\
\hline 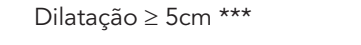 & 56 & 74,7 & 84 & 83,2 & 0,167 \\
\hline Sem presença de analgesia \# & 43 & 66,2 & 127 & 83,6 & 0,004 \\
\hline Na presença de analgesia \# & 82 & 77,4 & 13 & 76,5 & 0,575 \\
\hline Uso de analgesia \# & 149 & 54,4 & 21 & 7,7 & $<0,001$ \\
\hline Primíparas & 107 & 64,8 & 17 & 12,9 & $<0,001$ \\
\hline Multíparas & 42 & 38,5 & 4 & 3,0 & $<0,001$ \\
\hline Dilatação $<5 \mathrm{~cm} * \star \star$ & 84 & 63,2 & 11 & 12,4 & $<0,001$ \\
\hline 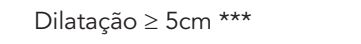 & 59 & 46,1 & 10 & 5,6 & $<0,001$ \\
\hline Sem presença de ocitocina & 20 & 18,2 & 6 & 3,7 & $<0,001$ \\
\hline Na presença de ocitocina & 127 & 48,6 & 15 & 13,9 & $<0,001$ \\
\hline Uso da episiotomia & 41 & 14,8 & 152 & 54,9 & $<0,001$ \\
\hline Primípara & 37 & 22,2 & 106 & 79,1 & $<0,001$ \\
\hline Multípara & 4 & 3,6 & 44 & 31,9 & $<0,001$ \\
\hline Assistência sem intervenção \#\# & 37 & 14,3 & 13 & 5,3 & $<0,001$ \\
\hline Tipo de parto & & & & & $<0,001$ \\
\hline Cesárea & 13 & 4,7 & 12 & 4,3 & \\
\hline Fórceps & 3 & 1,1 & 26 & 9,4 & \\
\hline Espontâneo & 261 & 94,2 & 239 & 86,3 & \\
\hline Apgar $<7$ \#\#\# & 1 & 0,4 & 1 & 0,4 & 0,990 \\
\hline Internação neonatal § & 6 & 2,2 & 14 & 5,1 & 0,07 \\
\hline
\end{tabular}

* O n amostral de cada variável não inclui seus valores ignorados;

** Método estatístico empregado: qui-quadrado de Pearson ou Fisher Exact;

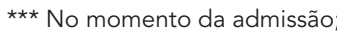

\# Analgesia peridural com dose única;

\# Inclui parto vaginal não instrumental, sem analgesia, ocitocina, amniotomia e episiotomia;

\#\#\# No 5o minuto de vida;

$\S$ Unidades de terapia intensiva e intermediária.

A presença predominante de métodos para aceleração do trabalho de parto, observada no mundo inteiro, se reflete na discussão sobre a definição do "parto normal" na literatura. Tanto o European Perinatal Health Report 19 quanto o Normal Birth Consense Statement 20, elaborado por entidades profissionais da Grã-Bretanha e o Joint Policy Statement on Normal Childbirth 21 das entidades canadenses definem o parto "normal" como aquele que se inicia sem medicação, termina como parto vaginal sem episiotomia e no qual não houve uso de instrumentos e de 
analgesia peridural, mas não exclui intervenções para acelerar o trabalho de parto e medicação oral ou intravenosa para o alívio da dor do parto. De acordo com essa definição, foram encontradas na Europa percentuais entre 26,2\% e 70\% de partos "normais" 19. Utilizando o indicador composto (parto vaginal não instrumental, sem analgesia, amniotomia, ocitocina e episiotomia), construído para este estudo, o CPN mostrou percentual menor com diferença significativa.

A taxa de analgesia peridural no HG foi um achado incomum para a assistência obstétrica no âmbito do SUS e até maior do que encontrada em outros países ${ }^{22}$. A analgesia é inexistente no CPN e no HP é oferecida de forma seletiva e restrita. Em contraponto, no HG, sua oferta para todas as mulheres faz parte da política institucional, desde que ela se tornou um direito no contexto da humanização da assistência ao parto e nascimento. Ainda que menor do que no $\mathrm{HG}$, o percentual da sua realização de $7,7 \%$ no HP é quase o dobro da taxa nacional estimada em 2006 7. A diferença importante na taxa de analgesia entre o SUS e o setor privado, de 3,5\% e 14,2\% 7, é sinal de desigualdade social na assistência ao parto e alvo de críticas justificadas. d'Orsi et al. 9 encontraram taxas muito baixas de analgesia peridural no parto vaginal - 3,1\% e 4,4\% - nas mulheres atendidas pelo SUS.

A aplicação em dose única permite o uso concomitante de práticas não farmacológicas do manejo da dor e talvez possa explicar a baixa proporção de parto a fórceps entre as mulheres do HG, apesar do uso liberal da analgesia. De La Chapelle et al. 23 encontraram resultados semelhantes ao comparar mulheres submetidas à analgesia peridural sem e com deambulação.

Segundo Diniz 2, a assistência ao parto normal no Brasil é excessivamente intervencionista e desnecessariamente traumática, tornando tanto a cesárea uma opção atraente como via do parto quanto a analgesia peridural uma demanda justificada. Apesar de o delineamento do estudo não permitir conclusões sobre uma possível associação entre uso de ocitocina e analgesia 3,14 , houve uso da medicação com maior frequência na presença da analgesia tanto no HG quanto no HP.

$\mathrm{O}$ uso frequente da analgesia peridural num serviço, que foi destacado pelo emprego de métodos não farmacológicos, aponta para uma contradição e o papel importante da atuação e das atitudes do profissional diante da demanda e da oferta de métodos de alívio da dor. A motivação das mulheres para a utilização da analgesia durante o trabalho de parto e o seu conhecimento sobre as vantagens e desvantagens desse método merecem maiores estudos. Parece que a disponi- bilidade de práticas assistenciais humanizadas não diminui a demanda por analgesia peridural, frente ao uso de intervenções no trabalho de parto e à oferta deliberada. Do outro lado, uma oferta menos seletiva para as mulheres atendidas pelo SUS deve ser considerada como prática que visa à humanização do parto.

A epísiotomia é outra intervenção com recomendação para que não seja realizada de rotina, contudo ainda é frequentemente utilizada 24 . No estudo de d'Orsi et al. 9, 77,7\% das mulheres do hospital público e $85,2 \%$ da maternidade conveniada foram submetidas à episiotomia. O presente estudo mostra uso com menor frequência no CPN e no HG onde a assistência é prestada por enfermeira obstetra, todavia o resultado do HP também está bem abaixo da média de 80,3\% 7 da Região Sudeste. Esse avanço pode ser fruto da discussão sistemática de práticas embasadas em evidências e da humanização da assistência ao parto e nascimento envolvendo os serviços e a Secretaria Municipal de Saúde de Belo Horizonte 25 . Nos sistemas de dados nacionais, não há qualquer informação sobre a taxa de episiotomia apesar de ser um indicador importante da qualidade assistencial 2.

Hatem et al. 15 mostram associação entre a assistência ao parto por obstetriz e baixas taxas de episiotomia e altas taxas de integridade perineal. De modo geral, a não realização rotineira da episiotomia é relacionada ao modelo assistencial humanizado e de baixa intervenção. Na Europa, a realização da episiotomia oscila entre 9,7\% na Dinamarca e $24,3 \%$ nos Países Baixos 19 . As taxas encontradas por Schneck 12, de 25,7\% num CPN em São Paulo e de 32,9\% num hospital com atuação predominante da enfermeira obstetra ao parto vaginal, sugerem também sua associação com o tipo de prestador da assistência.

Mesmo que métodos não farmacológicos como deambulação, banhos, massagens e liberdade de movimentos e a presença do acompanhante sejam indicadores importantes para o modelo assistencial humanizado, sua comparação, prevista no projeto de pesquisa, não foi possível. Embora a presença do acompanhante seja prática comprovadamente benéfica $26 \mathrm{e}$ rotineira nos hospitais de Belo Horizonte que atendem pelo SUS 25 , ela foi registrada somente no HG e no CPN. Por outro lado, para o uso de práticas não farmacológicas para o alivio da dor, que favorecem o trabalho de parto, não foram encontrados registros sistemáticos em nenhum modelo de atenção. A falta dos registros tanto da presença do acompanhante no HP quanto do uso de métodos não farmacológicos do alívio da dor no CPN e no HG, sugere que ambas as práticas não recebem a mesma atenção, por parte 
dos profissionais, que procedimentos invasivos. No contexto da humanização da assistência ao parto, a atenção ao registro dessas práticas é de grande importância para a avaliação de seu impacto sobre os resultados maternos e neonatais.

A baixa frequência do parto a fórceps no HG pode estar relacionada à assistência prestada por enfermeira obstetra como mostra revisão sistemática realizada por Hatem et al. 15 . Por outro lado, o fato de o HP ser local de residência médica pode ter influenciado seu uso maior. A taxa de cesárea encontrada no HP e no HG é baixa em ambos os modelos de atenção e sugere indicações apropriadas para as mulheres de risco habitual admitidas em trabalho de parto espontâneo ${ }^{3}$. Considerando a taxa geral de cesárea em 2006, dos dois serviços de 35\% e 26\% (Secretaria Municipal de Saúde de Belo Horizonte), respectivamente, as indicações para o parto cirúrgico nas gestações de risco, merecem estudos posteriores.

Por mais que não se encontrem diferenças significativas na proporção de recém-nascidos com Apgar menor do que 7 no 5 o minuto entre os três modelos assistenciais, os recém-nascidos foram internados em unidade neonatal com maior frequência no HP. Esse fato pode chamar atenção para a possibilidade de os recém-nascidos terem sidos submetidos a intervenções desnecessárias 27.

O CPN foi a modalidade assistencial com maior percentual de mulheres sem ocitocina, sem amniotomia e sem episiotomia, revelando o gradiente negativo do uso de intervenções entre os dois extremos, o CPN e o HP. O caso específico do HG não se enquadrou nesse construto teórico por causa do alto percentual de analgesia, que deve ter influenciado o uso da ocitocina. Entretanto, quando se utiliza o indicador composto, o gradiente negativo e a posição do HG entre os dois extremos aparecem.

Embora pareça que os riscos das intervenções sejam banalizados pelos profissionais nos três modelos assistenciais estudados e que haja resistência ao uso seletivo de intervenções no parto, os resultados apontam o CPN como lugar preferencial para a assistência ao parto das mulheres de risco habitual. Os resultados da assistência no CPN relativo ao índice de Apgar e à internação neonatal também reforçam as recomendações da Organização Mundial da Saúde sobre o lugar para o parto ${ }^{3}$. Tal perspectiva é disseminada em outros países, com base em estudos observacionais e experimentais sólidos 3,28,29,30. Sua implementação depende mais da vontade política do que da produção de evidências adicionais, uma vez que a mudança do modelo releva os conflitos de interesses estabelecidos e altera relações de poder existentes dos profissionais entre si e entre eles e as usuárias 2 .

Mesmo nas instituições com incorporação de práticas humanizadas, baseadas em evidências, a transformação do modelo assistencial na obstetrícia é um desafio atual e urgente que requer esforços tanto de gestores quanto de profissionais de saúde. 


\section{Resumo}

Estudo transversal com 831 gestantes, de risco habitual, sobre o manejo do trabalho de parto num Centro de Parto Normal (CPN), num hospital vencedor do título "Galba de Araújo" (HG) e numa maternidade com modelo assistencial prevalente (HP). O uso da ocitocina no CPN foi de 27,9\%, no HG 59,5\% e no HP 40,1\%, enquanto a amniotomia foi realizada em $67,6 \%, 73,6 \%$ e $82,2 \%$ das mulheres, respectivamente. A realização da episiotomia foi menor nas modalidades com incorporação de práticas humanizadas: 7,2\% no CPN e 14,8\% no $H G$ versus $54,9 \%$ no HP. A prática de oferta liberal no $H G$ resultou numa taxa de analgesia superior $(54,4 \%)$ à do HP $(7,7 \%)$. O percentual de internação dos recém-nascidos e o de parto a fórceps foram mais altas no HP, mas não houve diferenças para o índice de Apgar e para a taxa de cesárea. Os resultados sugerem resistência ao uso seletivo de intervenções em todos os modelos assistenciais, embora favoreçam o CPN como estratégia no controle das intervenções durante o trabalho de parto e parto nas gestantes de risco habitual sem prejuízos para as mulheres e os recém-nascidos.

Parto Humanizado; Trabalho de Parto; Saúde Materno-Infantil; Pesquisa sobre Serviços de Saúde

\section{Colaboradores}

S. E. Vogt participou substancialmente da realização da pesquisa, da coleta e análise dos dados e da redação e revisão do artigo. S. G. Diniz elaborou a pesquisa, participou substancialmente da sua realização e da análise dos dados, da redação e revisão do artigo. C. M. Tavares participou substancialmente da análise dos dados e da redação e revisão do artigo. N. C. P. Santos participou substancialmente da coleta e análise dos dados e da redação e revisão do artigo. C. A. Schneck participou substancialmente da elaboração e realização da pesquisa e da redação e revisão do artigo. B. Zorzam participou da realização da pesquisa, da coleta de dados e da redação do artigo. D. A. Vieira participou da pesquisa, da coleta dos dados e da redação do artigo. K. S. Silva e M. A. B. Dias participaram substancialmente da redação e revisão do artigo.

\section{Agradecimentos}

Ao Ministério da Saúde pelo financiamento.

\section{Referências}

1. Santos ML. Humanização da assistência ao parto e nascimento: um modelo teórico [Dissertação de Mestrado]. Florianópolis: Centro de Ciências da Saúde, Universidade Federal de Santa Catarina; 2002.

2. Diniz SG. Gênero, saúde materna e o paradoxo perinatal. Rev Bras Crescimento Desenvolv Hum 2009; 19:313-26.

3. Enkin M, Keirse MJNC, Renfrew M, Neilson J. A guide to effective care in pregnancy \& childbirth. Oxford: Oxford Medical Publications; 2005.

4. Silveira MF, Santos IS, Barros AJD, Matijasevich A, Barros FC, Victora CG. Aumento da prematuridade no Brasil: revisão de estudos de base populacional: revisão. Rev Saúde Pública 2008; 42:957-64.

5. Victora CG, Aquino EML, Leal MC, Monteiro CA, Barros FC, Szwarcwald CL. Saúde de mães e crianças no Brasil: progressos e desafios. http://down load.thelancet.com/flatcontentassets/pdfs/bra zil/brazilpor2.pdf (acessado em 10/Nov/2010).

6. Wagner M. Fish can't see water: the need to humanize birth. Int J Gynaecol Obstet 2001; 75 Suppl 1:S25-37.

7. Ministério da Saúde. Pesquisa Nacional de Demografia e Saúde da Criança e da Mulher - PNDS 2006: dimensões do processo reprodutivo e da saúde da criança. Brasília: Ministério da Saúde; 2009.

8. Oliveira MIC, Dias MAB, Cunha CB, Leal MC. Qualidade da assistência ao trabalho de parto pelo Sistema Único de Saúde, Rio de Janeiro (RJ), 19992001. Rev Saúde Pública 2008; 42:895-902. 
9. d'Orsi E, Chor D, Giffin K, Angulo-Tuesta A, Barbosa GP, Gama AS, et al. Qualidade da atenção ao parto em maternidades do Rio de Janeiro, Brasil. Rev Saúde Pública 2005; 39:646-54.

10. Ministério da Saúde. Parto, aborto e puerpério: assistência humanizada à saúde. Brasília: Ministério da Saúde; 2001.

11. Rattner D. Humanização na atenção a nascimentos e partos: ponderações sobre políticas públicas. Interface Comun Saúde Educ 2009; 13 Suppl 1:S759-68.

12. Schneck CA. Estudo comparativo dos resultados maternos e perinatais em Centro de Parto Normal peri-hospitalar e hospitalar [Tese de Doutorado]. São Paulo: Escola de Enfermagem, Universidade de São Paulo; 2009.

13. Simpson KR. Perinatal patient safety and quality. J Perinat Neonatal Nurs 2011; 25:103-7

14. Tracy SK, Sullivan E, Wang YA, Black D, Tracy M. Birth outcomes associated with interventions in labour amongst low risk women: a populationbased study. Women Birth 2007; 20:41-8.

15. Hatem M, Sandall J, Devane D, Soltani H, Gates S. Midwife-led versus other models of care for childbearing women. Cochrane Database Syst Rev 2008; (04):CD004667.

16. Sakae TM, Freitas PF, d'Orsi E. Fatores associados a taxas de cesárea em hospital universitário. Rev Saúde Pública 2009; 43:472-80

17. Clark SL, Simpson KR, Knox GE, Garite TJ. Oxytocin: new perspectives on an old drug. Am J Obstet Gynecol 2009; 200:35.e1-6.

18. Smyth RMD, Alldred SK, Markham C. Amniotomy for shortening spontaneous labour. Cochrane Database Syst Rev 2007; (4):CD006167.

19. Euro-Peristat Project/Surveillance of Cerebral Palsy in Europe/European Surveillance of Congenital Anomalies/The European Information System to Monitor Short and Long-Term Morbidity to Improve Quality of Care and Patient Safety for VeryLow-Birth-Weight Infants. Perinatal health report 2008. http://www.europeristat.com/bm.doc/eu ropean-perinatal-health-report.pdf (acessado em 01/Nov/2010).
20. Making normal birth a reality. Consensus statement from the Maternity Care Working Party our shared views about the need to recognise, facilitate and audit normal birth. http://www.rsfq. qc.ca/normal_birth_consensus.pdf (acessado em 01/Nov/2010).

21. Joint policy statement on normal childbirth. J Obstet Gynaecol Can 2008; 30:1163-5.

22. Wiegers TA. The quality of maternity care services as experienced by women in the Netherlands. BMC Pregnancy and Childbirth 2009; 9:18.

23. de la Chapelle A, Carles M, Gleize V, Dellamonica J, Lallia A, Bongain A, et al. Impact of walking with epidural analgesia on obstetric outcomes of nulliparous women in spontaneous labour. Int J Obstet Anesth 2006; 15:104-8.

24. Carroli G, Mignini L. Episiotomy for vaginal birth. Cochrane Database Syst Rev 2009; (1):CD000081.

25. Maia MB. Humanização do parto: política pública, comportamento organizacional e ethos profissional na rede pública e privada de Belo Horizonte [Dissertação de Mestrado]. Belo Horizonte: Programa de Pós-graduação em Ciências Sociais, Pontifícia Universidade Católica de Minas Gerais; 2008.

26. Bruggemann OM, Parpinelli MA, Osis MJD. Evidências sobre o suporte durante o trabalho de parto/parto: uma revisão da literatura. Cad Saúde Pública 2005; 21:1316-27.

27. Albers LL. Overtreatment of normal childbirth in US hospitals. Birth 2005; 32:67-8.

28. Gottvall K, Waldenström U, Tingstig C, Grunewald C. In-hospital birth center with the same medical guidelines as standard care: a comparative study of obstetric interventions and outcomes. Birth 2011; 38:120-8.

29. Davis D, Baddock S, Pairman S, Hunter M, Benn C, Wilson D, et al. Planned place of birth in New Zealand: does it affect mode of birth and intervention rates among low-risk women? Birth 2011; 38:111-9.

30. Hodnet ED, Downe S, Walsh D, Weston J. Alternative versus conventional institutional settings for birth. Cochrane Database Syst Rev 2010; (9):CD000012.

Recebido em 27/Jan/2011

Versão final reapresentada em 20/Jun/2011

Aprovado em 27/Jun/2011 